\title{
Pseudoarachnoiditis in Spontaneous Intracranial Hypotension
}

\author{
Özlem Alkan¹, Tülin Yıldırım¹, Osman Kızılkılıç², Sait Albayram², Naime Altınkaya \\ ${ }^{1}$ Department of Radiology, Faculty of Medicine, Başkent University, Ankara, Turkey \\ ${ }^{2}$ Department of Radiology, Cerrahpaşa Faculty of Medicine, İstanbul University, İstanbul, Turkey
}

\begin{abstract}
Spontaneous intracranial hypotension is an important cause of new daily persistent headaches in young and middle-aged individuals. The diagnosis is made based on low cerebrospinal fluid pressure with characteristic findings upon brain and spinal magnetic resonance imaging (MRI). We present the case of a 15-year-old boy with spontaneous intracranial hypotension. Although his brain MRI was normal, his lumbar spinal MRI showed clustering of the nerve roots characteristic of arachnoiditis. Radionuclide cisternography revealed an epidural leak, which was treated with an epidural blood patch. The patient reached a near-full recovery within $24 \mathrm{~h}$, and the lumbar spinal MRI findings mimicking arachnoiditis disappeared.
\end{abstract}

Key Words: Spontaneous intracranial hypotension, pseudoarachnoiditis, spinal MRI

Received: 27.02.2009 Accepted: 12.03.2009

\section{Introduction}

The most common causes of intracranial hypotension include persistent cerebrospinal fluid (CSF) leakage from lumbar puncture, spontaneous CSF leakage, or excessive CSF drainage. Spontaneous intracranial hypotension $(\mathrm{SIH})$ is defined as a syndrome of reduced CSF pressure that occurs in the absence of dural puncture, surgery, or trauma. The pathogenesis is generally thought to be an occult leak of CSF through small defects in the meninges, with a resultant decrease in CSF volume and pressure (1). Intracranial hypotension typically presents with postural headaches. Typical findings on brain magnetic resonance imaging (MRI) are diffuse pachymeningeal enhancement, subdural fluid collections, pituitary hyperemia, engorgement of venous structures, and brain sagging, although brain MRI scans are sometimes normal (1). Spinal imaging is usually recommended if brain MRI yields normal findings or if treatments fail.

Although numerous spinal MRI findings of SIH have been described (2), we report a novel case of SIH in which the brain MRI scan was normal, but the lumbar spinal MRI revealed clustering of the cauda equina fibers mimicking arachnoiditis.

\section{Case Report}

A previously healthy 15 -year-old boy developed a suddenonset headache associated with blurred vision. The headache was aggravated by sitting or standing and was relieved by lying down. He had no history of dural tear, such as lumbar puncture, head or spinal trauma, or surgical drainage. His neurological and physical examinations revealed no abnor- malities, and brain MRI and magnetic resonance venography yielded normal results. Spinal MRI without contrast enhancement revealed clumps of adherent roots centrally located within the thecal sac (Figure $1 \mathrm{~A}, \mathrm{~B}$ ).

A lumbar puncture subsequently revealed a depressed opening CSF pressure of $40 \mathrm{mM} \mathrm{H}_{2} \mathrm{O}$, but CSF studies disclosed no other abnormalities. Radionuclide cisternography showed bilateral, extradural tracer accumulation at the lumbar spine. Since the patient did not respond to analgesics, a lumbar epidural blood patch was performed. About $10 \mathrm{~cm}^{3}$ of autologous blood was given at the L3-L4 level. The patient's symptoms improved to almost complete resolution within 24 $h$, and the arachnoiditis-like spinal MRI findings completely resolved (Figure $2 \mathrm{~A}, \mathrm{~B}$ ).

\section{Discussion}

The most common clinical manifestation of $\mathrm{SIH}$ is an orthostatic headache that may occur within seconds to minutes of assuming the upright position. Associated symptoms are common and include nausea, vomiting, dizziness, diplopia, photophobia, hearing impairment, neck pain, and blurred vision. Typically, the CSF opening pressure is $<6 \mathrm{~cm}$ of $\mathrm{H}_{2} \mathrm{O}$; it can also be immeasurable, negative, or even normal. The CSF may appear normal upon examination, or may reveal xanthochromia, lymphocytic pleocystosis, or elevated protein content (1). A primary clinical component of the new diagnostic criteria is improvement of symptoms after epidural blood patching (3). In the case described here, the typical clinical presentation and complete resolution of clinical and radiological signs after epidural blood patching supported the diagnosis of $\mathrm{SIH}$. 


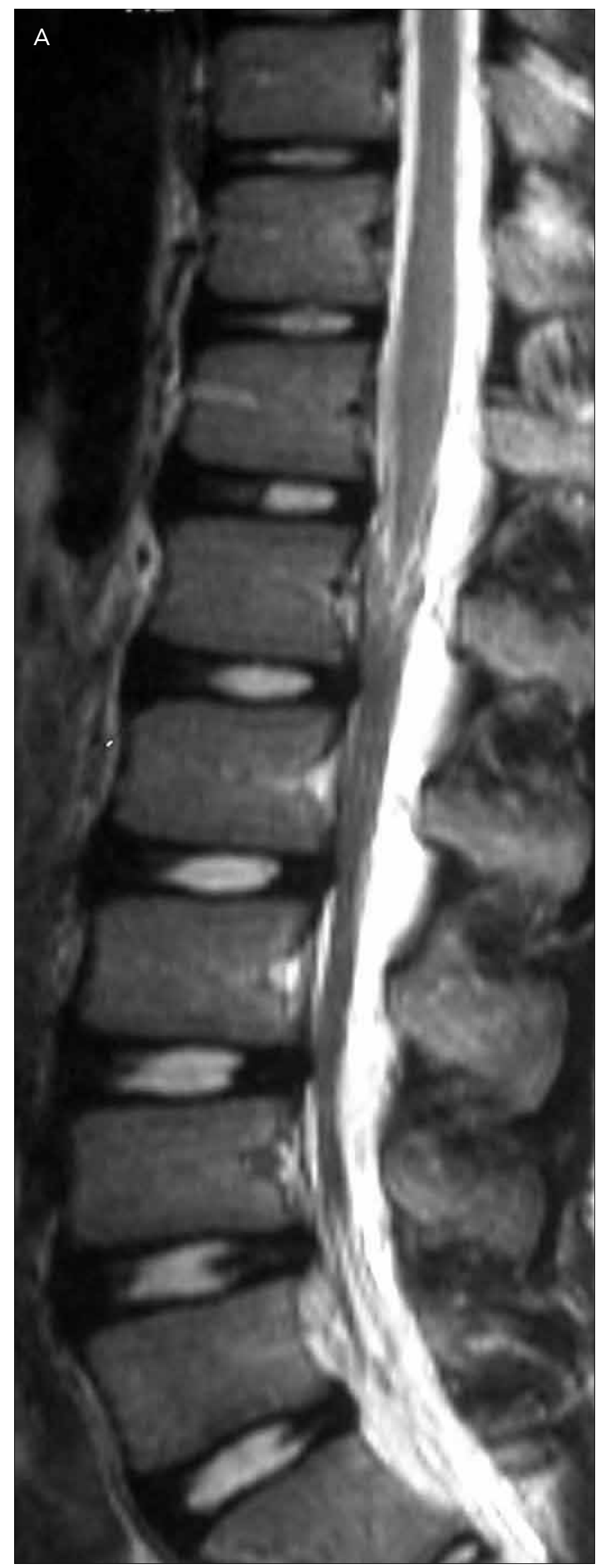

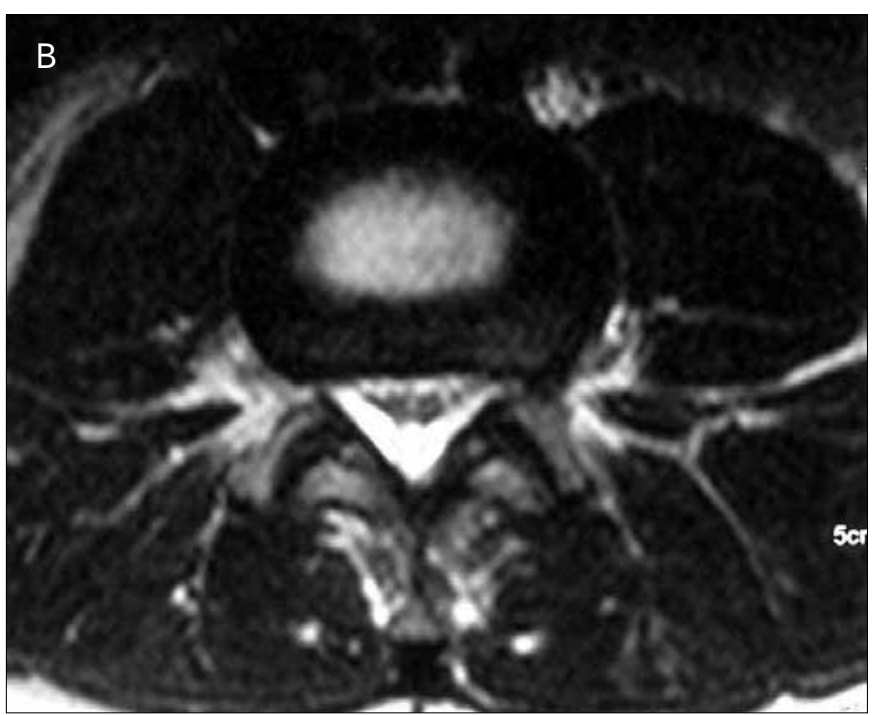

Figure 1. Sagittal (A) and axial (B) T2-weighted images revealing clumps of adherent roots centrally located within the thecal sac

Most of the MRI features of SIH can be explained as compensatory changes related to the loss of CSF volume. According to the Monroe-Kellie hypothesis, the sum of the volumes of intracranial blood, CSF, and cerebral tissue must remain constant in an intact cranium (4). A decrease in CSF volume will therefore lead to an increase in the volume of other compartments. An increase in the vascular component can compensate for the loss of CSF from the spine and can lead to pachymeningeal enhancement, engorgement of venous structures, and pituitary hyperemia. Alternatively, an increase in the intracranial CSF component can lead to subdural hygromas. Brain MRI scans are normal in up to $20 \%$ of patients with $\mathrm{SIH}$ (5). In the absence of brain MRI abnormalities, spinal MRI is recommended to confirm the SIH diagnosis in patients (2).

Given the connection between the intracranial and intraspinal volumes, the Monro-Kellie hypothesis should apply to the spinal canal. Decreased intrathecal CSF leads to collapse of the spinal subarachnoid space and results in the enlargement of the epidural space; this phenomenon is associated with the enlargement of epidural veins and even accumulation of epidural CSF in the spinal canal (2). In addition to the Monro-Kellie hypothesis, a unique phenomenon ruling the spinal presentations of SIH is leakage of CSF into the epidural space (6). Spinal MR imaging in SIH usually reveals a collapsed dural sac, extradural fluid collections, spinal meningeal enhancement, and dilatation of the epidural venous plexus, meningeal diverticula, and the presence of retrospinal fluid collections at C1-C2 $(2,7)$. The collapsed dural sac has a characteristic hexagon-like contour, which has been described as a "festooned appearance" (2).

Arachnoiditis is an inflammation of the arachnoid mater. This inflammation may cause fibrinous exudates around the roots that cause them to adhere to each other and/or the thecal sac. The causes of spinal arachnoiditis are varied and include infection, intrathecal steroids or anesthetic agents, trauma, surgery, and intrathecal hemorrhage (8). MRI can be 

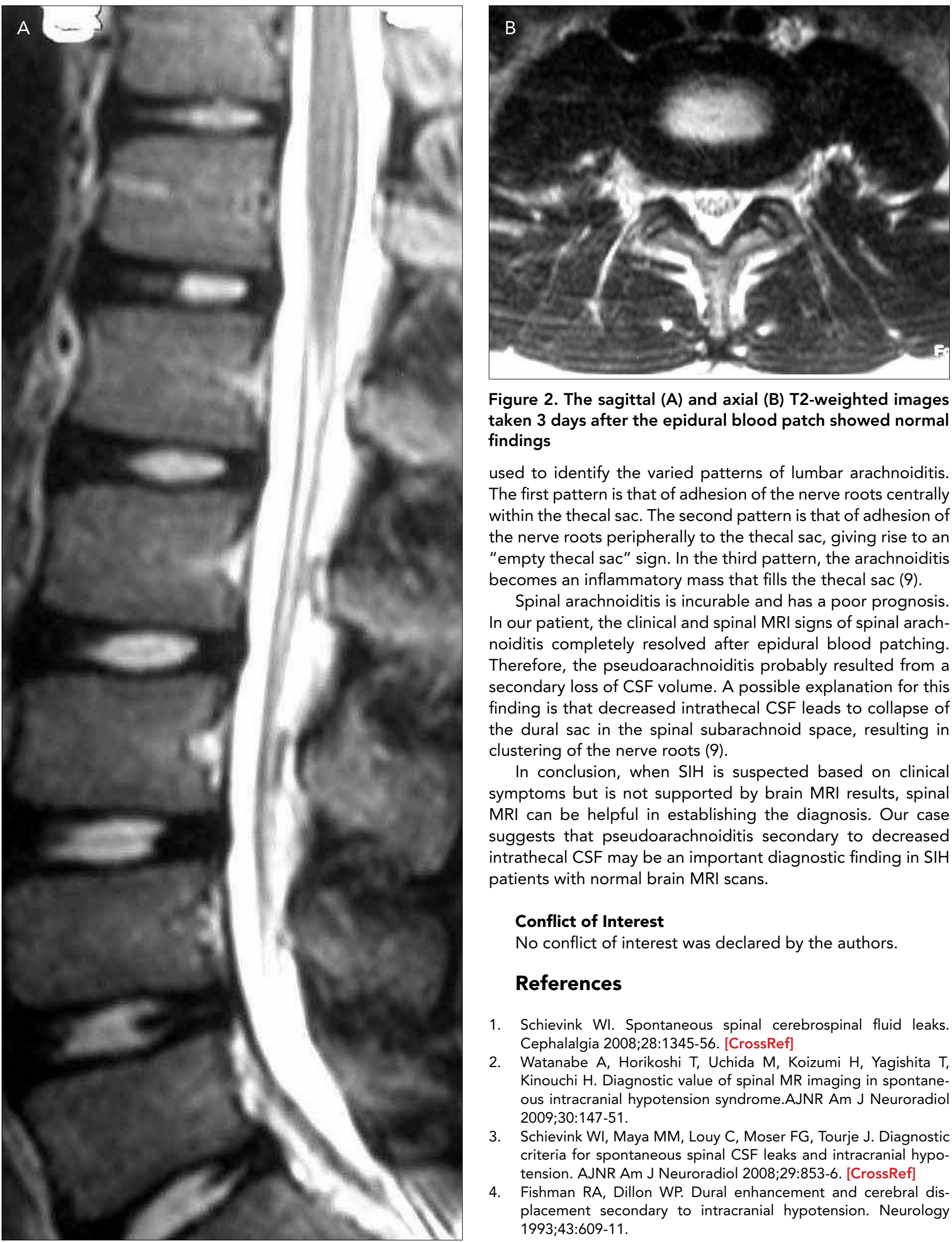

Figure 2. The sagittal (A) and axial (B) T2-weighted images taken 3 days after the epidural blood patch showed normal findings

used to identify the varied patterns of lumbar arachnoiditis. The first pattern is that of adhesion of the nerve roots centrally within the thecal sac. The second pattern is that of adhesion of the nerve roots peripherally to the thecal sac, giving rise to an "empty thecal sac" sign. In the third pattern, the arachnoiditis becomes an inflammatory mass that fills the thecal sac (9).

Spinal arachnoiditis is incurable and has a poor prognosis. In our patient, the clinical and spinal MRI signs of spinal arachnoiditis completely resolved after epidural blood patching. Therefore, the pseudoarachnoiditis probably resulted from a secondary loss of CSF volume. A possible explanation for this finding is that decreased intrathecal CSF leads to collapse of the dural sac in the spinal subarachnoid space, resulting in clustering of the nerve roots (9).

In conclusion, when $\mathrm{SIH}$ is suspected based on clinical symptoms but is not supported by brain MRI results, spinal MRI can be helpful in establishing the diagnosis. Our case suggests that pseudoarachnoiditis secondary to decreased intrathecal CSF may be an important diagnostic finding in $\mathrm{SIH}$ patients with normal brain MRI scans.

\section{Conflict of Interest}

No conflict of interest was declared by the authors.

\section{References}

1. Schievink WI. Spontaneous spinal cerebrospinal fluid leaks. Cephalalgia 2008;28:1345-56. [CrossRef]

2. Watanabe A, Horikoshi $T$, Uchida $M$, Koizumi $H$, Yagishita $T$, Kinouchi $H$. Diagnostic value of spinal MR imaging in spontaneous intracranial hypotension syndrome.AJNR Am J Neuroradiol 2009;30:147-51.

3. Schievink WI, Maya MM, Louy C, Moser FG, Tourje J. Diagnostic criteria for spontaneous spinal CSF leaks and intracranial hypotension. AJNR Am J Neuroradiol 2008;29:853-6. [CrossRef]

4. Fishman RA, Dillon WP. Dural enhancement and cerebral displacement secondary to intracranial hypotension. Neurology 1993;43:609-11. 
5. Schievink WI. Spontaneous spinal cerebrospinal fluid leaks and intracranial hypotension. JAMA 2006;295:2286-96. [CrossRef]

6. Chen CJ, Lee TH, Hsu HL, Tseng YC, Wong YC, Wang LJ. Spinal MR findings in spontaneous intracranial hypotension. Neuroradiology 2002;44:996-1003.

7. Yousry I, Förderreuther S, Moriggl B, Holtmannspötter M, Naidich TP, Straube A et al. Cervical MR imaging in postural head- ache: MR signs and pathophysiological implications. AJNR Am J Neuroradiol 2001;22:1239-50.

8. Ross JS, Masaryk TJ, Modic MT, Delamater R, Bohlman H, Wilbur $G$ et al. MR imaging of lumbar arachnoiditis. AJR Am J Roentgenol 1987;149:1025-32.

9. Delamarter RB, Ross JS, Masaryk TJ, Modic MT, Bohlman HH. Diagnosis of lumbar arachnoiditis by magnetic resonance imaging. Spine 1990;15:304-10. [CrossRef] 